\title{
Evaluation of the graded redefined assessment of strength, sensibility and prehension (GRASSP) in children with tetraplegia
}

\author{
MJ Mulcahey ${ }^{1,2} \cdot$ Christina Calhoun Thielen $^{1} \cdot$ Kathryn Dent $^{1} \cdot$ Rebecca Sinko $^{1} \cdot$ Cristina Sadowsky $^{3}$. \\ Rebecca Martin ${ }^{3} \cdot$ Lawrence C. Vogel $^{4} \cdot$ Loren Davidson $^{5} \cdot$ Heather Taylor $^{6} \cdot$ Jackie Bultman $^{7} \cdot$ John Gaughan $^{1}$
}

Received: 10 October 2017 / Accepted: 17 February 2018 / Published online: 26 March 2018

(c) International Spinal Cord Society 2018

\begin{abstract}
Study design Psychometric study.

Objective To validate the GRASSP in pediatric SCI populations and establish the lower age of test administration. Setting United States: Pennsylvania, Maryland, Illinois, Michigan, California, Texas.

Methods Mean, SD and range of scores were calculated and examined for known-group differences. Test-retest reliability was measured by the intra-class correlation, concurrent validity of the GRASSP against the SCIM, SCIM-SS, and the CUEQ was measured by the Spearman correlation.

Results GRASSP scores differed between participants with motor complete and incomplete injuries $(p=<0.0001-0.036)$. Test-retest reliability was strong (ICC $=0.99)$. Weak correlation with the total SCIM $(r=0.33-0.66)$, and moderate to strong correlation with the SCIM-SC $(r=37-0.70)$ and CUE-Q $(r=0.40-0.84)$.

Conclusion Results support the validity of the GRASSP and provide evidence that the scores are reliable when administered to children. The GRASSP sensory and strength subtests are recommended for children beginning at 6 years of age, and the GRASSP prehension performance/ability subtest for children beginning at 8 years of age. Normative data are needed for the performance components of the GRASSP.
\end{abstract}

\section{Introduction}

The Graded Redefined Assessment of Strength, Sensibility and Prehension (GRASSP) was developed to address the void in the availability of SCI measures that detect subtle and important changes in sensation and motor impairments and functional capacity of the hand [1]. The GRASSP uses well-established methods to evaluate dorsal and palmar sensation [2] and manual muscle strength [3]. The

MJ Mulcahey

maryjane.mulcahey@jefferson.edu

Thomas Jefferson University, Philadelphia, PA, USA

Shriners Hospitals for Children, Philadelphia, PA, USA

3 Kennedy Krieger Rehabilitation Institute, Baltimore, MD, USA

4 Shriners Hospitals for Children, Chicago, IL, USA

5 Shriners Hospitals for Children, Sacramento, CA, USA

6 TIRR Herman Memorial, Houston, TX, USA

7 Mary Free Bed Rehabilitation Hospital, Grand Rapids, MI, USA
GRASSP prehension domain scores the ability to generate three common grasp patterns (key, palmar, tip-to-tip) and draws upon concepts from other prehension performancebased measures designed for persons with tetraplegia [4]. Psychometric properties of the GRASSP with adult samples are strong, with moderate-to-high inter- and intra-rater reliability and good concurrent validity with the Spinal Cord Independence Measure (SCIM)-III [5, 6]. The GRASSP also discriminates across neurological levels of known differences with a high degree of reliability [6], can be used to predict neurological recovery $[7,8]$, and is able to detect small but meaningful changes as a result of recovery and intervention $[9,10]$. The strong psychometric properties of the GRASSP have led to its increasing acceptance in clinical practice and use in clinical trials to evaluate primary endpoints related to hand function [11-14].

Studies to establish and validate meaningful and responsive outcome instruments are paramount to research and practice, although efforts such as the development of the GRASSP often neglect the pediatric SCI population. For example, although cervical spinal cord injuries (cSCI) in 
children and adults result in similar upper extremity impairments [15], and even though children and adults receive similar interventions to improve hand function [1619], the psychometric properties of the GRASSP have not been established in youth with SCI [5]. Given the strong psychometric properties in adult samples and its increase use in cSCI clinical trials, it is important to evaluate the GRASSP in children with cSCI. With established psychometric properties of the GRASSP, potential barriers to children's participation in clinical trials associated with measurement could be reduced and practice patterns associated with the use of outcomes instruments that have little or no psychometric support in children could be addressed. Thus, the purpose of this study was threefold. The first purpose was to determine the lower age in which the GRASSP has utility. Second, test-rest reliability was evaluated. Last, known-group and concurrent validity of the GRASSP was examined. We hypothesized that the GRASSP would demonstrate the following psychometric properties: (1) good known-group validity as evidenced by a statistically significant difference in mean scores among groups with known differences in hand function; (2) good test-retest reliability on repeated administration as evidenced by ICC values $=>0.85$, and; (3) good concurrent validity of the GRASSP subset scores when correlated with total SCIM-III, SCIM-III self-care subscale, and Capabilities of the Upper Extremity Questionnaire (CUE-Q) as evidenced by correlation coefficients $=>0.75$. We hypothesized that very young children may not be able to complete the GRASSP due to inability to understand and follow test instructions, the inability to maintain attention for the duration of the test, and an inability to grasp and lift larger standardized test objects due to small hand size.

\section{Methods}

\section{Study design}

This study was part of a larger multi-center repeated measures study aimed to examine the psychometric properties of instruments used for adult SCI clinical trials in children and youths. The study was approved by each of the seven participating sites ethics/IRB committees.

\section{Participants}

A convenience sample of children with tetraplegia was recruited from 7 facilities across the United States specializing in pediatric rehabilitation and SCI care. Children were recruited at the point-of-usual, routine care for surveillance of SCI. Children were included if they were under 18 years of age, had cSCI as a result of a traumatic or non-traumatic acquired SCI with no change in neurological status for at least 3 months prior to study enrollment based on medical record documentation, and had the ability to maintain sitting balance, aided or unaided by support of a seating system or other mechanism. Children were excluded if they had no motor function except neck rotation, extension and flexion, progressive spinal cord disease, spina bifida, were in the immediate post-operative or rehabilitation phase of upper extremity tendon or nerve transfers, had a traumatic brain injury that interfered with the ability to comprehend and/or follow test instructions and directions, a suspected conversion syndrome or were suicidal. Child assent and parental consent were obtained. All applicable institutional and governmental regulations concerning the use of human participants and informed consent were followed during the course of this study.

\section{Demographic data}

A study specific case report form (CRF), using the National Institute of Neurological Disease and Stroke (NINDS) common data elements (CDE) [20] was used to record month and year of birth, gender, ethnicity, race, primary language, hand dominance, date of injury, cause of injury, type of schooling, and the child's highest education level. Demographic data were obtained from the medical record and/or through interview with the participant and/or caregiver.

\section{Neurological data}

The International Standards for Neurological Classification of SCI (ISNCSCI) [3] was used to determine severity, neurological level (NL) and American Spinal Injury Association Impairment Scale (AIS) Classification. Given that participants had to be neurologically stable for a period of three months, we obtained the ISNCSCI information from the medical records for youth who had the examinations within a year of study, but after neurological stability was documented. If the ISNCSCI examinations were performed $>12$ months prior to enrollment, they were completed again for children 6 years of age and older with the ability to participate in the examinations, at the time of enrollment.

\section{GRASSP}

The GRASSP is comprised of five subtests designed to measure three integrated domains of sensorimotor function of the hand: sensibility, strength and prehension [6]. Palmar sensation and dorsal sensation are measured by conducting the Semmes Weinstein Monofilament Testing (SWM) [2]. The monofilaments are each placed at three standardized surface locations on the palmar and dorsal aspects of the 
hand, and grams of force are recorded on a four-point scale, where no response to filament testing $=0$, response to 6.65 filament testing $=1$, response to filament testing $4.56=2$, response to filament testing $4.31=3$, and response to filament testing $3.61=4$. Test locations are scored for both the palmar and dorsal aspects of both the left and right hands. Scores from the right and left dorsal and palmar locations are summed, to yield dorsal and palmar sensation subtest scores respectively, each ranging between 0 and 12 .

The strength of 10 muscles in each arm is evaluated using standard manual muscle testing techniques, and scored using a traditional scale between 0 (flaccid, no movement) and 5 (full range of motion, with maximal resistance for age). The scores of the ten muscles are summed for each arm, yielding a total strength score between 0 and 50 for each limb. For this study, the amount of resistance applied during manual muscle testing of young children was less than what is typically applied when testing adults, considering the age, growth, and development of the children.

The prehension domain encompasses both ability and performance. Prehension ability (qualitative) measures the ability to generate a cylindrical grasp, and key and tip-to-tip pinch. Ability is observed, and scored on a scale between 0 (no ability to use wrist, thumb, fingers) to 4 (able to maintain wrist stability and utilize finger and thumb). The ability scores of each pattern are summed to yield a subtest prehension ability score for the right and left hands, with the total possible score for each hand $=12$. The test for prehension performance (quantitative) involves performance of six unilateral tasks. Each task is scored on a scale between 0 (unable) to 5 (fully able), with a total possible score of 30 for each hand. A time limit for each item is set $(75 \mathrm{~s})$. The scores from each GRASSP subtest are interpreted separately. A total GRASSP score is not calculated [6], but domains are unilaterally added for a total right score and total left score.

The GRASSP was administered twice by a trained physical or occupational therapist within $24 \mathrm{~h}$, with at least one hour between the first and second administration. The 24-h time frame was chosen to accommodate participants' schedules and to reduce the risk of missing the second administration. Scores were recorded on the GRASSP standardized scoring sheet. During GRASSP administration, adaptive equipment, functional hand splints, orthoses and neuroprostheses were not used. Any modifications or deviations from standardized GRASSP administration were recorded, and comments about challenges with data collection were noted, where applicable.

\section{Spinal Cord Independence Measure-III (SCIM-III)}

The SCIM-III [21] is a 19-item disease specific scale that measures daily activities of high relevance to persons with
SCI. The SCIM-III generates a total score between 0 and 100, where higher scores reflect greater level of physical function, and scores for three subscales are obtained: self care (score range between 0 and 20), respiration and sphincter (score range between 0-40), and mobility (inroom mobility score range between 0 and 10 ; indooroutdoor mobility score range between 0 and 30). Psychometric properties of the SCIM-III in children are strong [22]. We administered the SCIM-III to children using a combination of observation and interview; if needed, parents assisted in the interview. The SCIM-III total and self care subscales were used as comparator scores, as previously done to establish concurrent validity of the GRASSP in adults [5].

\section{Capabilities of the Upper Extremity Questionnaire (CUE-Q)}

The Capabilities of Upper Extremity-Questionnaire version 2.1 (CUE-Q) is a 17-item scale that is used to evaluate perceived difficulties or limitations associated with using the upper limbs to complete everyday activities [23]. The right and left limbs are evaluated and scored separately on a scale ranging between 0 (unable/complete difficulty) to 4 (no difficulty). The CUE-Q was used as a comparator measure to establish validity, as done to establish validity of the GRASSP with the adult sample [5]. In adult studies, the CUE-Q version 2.1 has strong concurrent validity (FIM Instrument ${ }^{\bullet}$ and ISNCSCI upper extremity motor score) and is responsive to change over time [23]. There are no previous pediatric studies on the CUE-Q.

\section{Investigator and rater training}

A two-day meeting, prior to the start of data collection was conducted at the lead site for all site investigators and site coordinators. During this meeting, site coordinators were trained in the study data collection, data management and data transmission protocols. To ensure fidelity in administration, scoring and interpretation, each site was provided with on-site training by the GRASSP developer, and was provided with the GRASSP version 1.0 tool kit which contains a comprehensive manual with test instructions and video demonstrations. Each research coordinator was also provided with a study procedural manual which contained standardized CRFs for recording data, and study specific procedural guidelines including those for de-identifying and transmission of data to the lead site. During the period of open enrollment and data collection, bimonthly team meetings were conducted via teleconference to discuss enrollment, study procedures, and to answer questions and provide clarity on data collection, management or other issues. 


\section{Data collection and management procedures}

Using site and participant specific unique identification numbers, data were de-identified prior to transmission to the lead site. Once received by the lead site, the study coordinator reviewed data for completeness and quality. If data were incomplete or if quality was poor, they were returned to the originating site with questions about accuracy and completeness. Following quality review, data were entered into a secure study specific database.

\section{Analysis}

Mean, standard deviation (SD), and range (R) values were calculated for each GRASSP subtest score for both the right and the left hands for the entire sample and as a function of AIS, age groups $(3-5 ; 6-12 ; 13-15 ; 16-17)$, and level of injury. Results of the entire sample were examined with reference to published results in adults [5].

Differences in GRASSP subtest scores between motor complete (AIS A+B) and motor incomplete (AIS C+D) categories were examined for evidence of known-group validity using ANOVA on ranks. We examined construct validity by evaluating the association between hand strength and prehension performance using a Pearson correlation coefficient ( $r$ ). Concurrent validity was assessed by the Spearman rank correlation coefficient $\left(\mathrm{r}_{\mathrm{s}}\right)$ of the bilateral summations of the GRASSP subtest scores for strength, sensation, and prehension performance against the SCIM-III total score, SCIM-III SC subscale score, and the CUE-Q total scores respectively. Results of the analyses were examined with reference to published studies in adults [5].

Intra-class correlations (ICC $[2,1]$ ) with $95 \%$ confidence intervals (CI) were calculated to examine test-retest reliability of GRASSP subtest scores for each upper limb. Testretest reliability was also examined for each age group, AIS classification, and NL groups.

The lower age limit in which each GRASSP subtest has utility was established by both qualitative and quantitative methods. Individual screen failures and participants in the youngest age group (3-5) were further analyzed by age. The proportion of individuals able to complete each test was evaluated. If an individual in this age group was unable to perform an assessment, the rater's comments were examined to determine if inability was age-related. Analyses were carried out using SPSS v24.0 (IBM Corp, Armonk, NY) and SAS v9.4 (SAS Institute, Cary, NC).

\section{Results}

A total of 64 children with tetraplegia were screened for participation, and 17 were not enrolled. Reasons for
Table 1 Sample Characteristics for GRASSP Trial 1 and Trial 2

\begin{tabular}{|c|c|c|}
\hline & Trial $1(N=47)$ & Trial $2(N=35)$ \\
\hline \multicolumn{3}{|c|}{ Age Group in Years $N(\%)$} \\
\hline $30-5$ & $5(11)$ & $3(9)$ \\
\hline $6-12$ & $15(32)$ & $10(29)$ \\
\hline $13-15$ & $12(26)$ & $11(31)$ \\
\hline $16-17$ & $15(32)$ & $11(31)$ \\
\hline \multicolumn{3}{|l|}{ Gender $N(\%)$} \\
\hline Male & $28(60)$ & $22(63)$ \\
\hline Female & $19(40)$ & $13(37)$ \\
\hline \multicolumn{3}{|l|}{ Race $N(\%)$} \\
\hline Caucasian & $41(87)$ & $29(83)$ \\
\hline Asian & $1(2)$ & $1(3)$ \\
\hline African American & $3(6)$ & $3(9)$ \\
\hline Other & $2(4)$ & $2(6)$ \\
\hline \multicolumn{3}{|l|}{ Ethnicity $N(\%)$} \\
\hline Hispanic & $9(19)$ & $8(23)$ \\
\hline Non-Hispanic & $38(81)$ & $27(77)$ \\
\hline \multicolumn{3}{|l|}{ NL grouping $N(\%)$} \\
\hline $\mathrm{C} 1-5$ & $23(49)$ & $19(54)$ \\
\hline C6 & $5(11)$ & $3(9)$ \\
\hline C7-T1 & $8(17)$ & $5(14)$ \\
\hline Unknown & $11(23)$ & $8(23)$ \\
\hline \multicolumn{3}{|c|}{ AIS classification $N(\%)$} \\
\hline $\mathrm{A}$ & $14(30)$ & $10(29)$ \\
\hline $\mathrm{B}$ & $4(9)$ & $4(11)$ \\
\hline $\mathrm{C}$ & $10(21)$ & $7(20)$ \\
\hline $\mathrm{D}$ & $8(17)$ & $6(17)$ \\
\hline Unknown & $11(23)$ & $8(23)$ \\
\hline
\end{tabular}

$N$ number, $N L$ neurological groupings, AIS American Spinal Injury Association Impairment Scale

exclusion included: one (6\%) had a legal guardian who was unable to give consent due to not speaking and understanding English, 4 (24\%) had complete injuries without any movement below the neck, $5(29 \%)$ were unable to follow instructions due to young developmental age (18 months - 5 years), and 6 (35\%) declined participation. As summarized in Table 1, the remaining 47 participants were between 3 and 17 years of age with chronic SCI. The majority of the participants were white (87\%), boys (59\%), and identified as non-Hispanic (81\%). ISNCSCI were missing in $11(23 \%)$ participants in trial one, and $8(23 \%)$ participants in trial two. Of the 47 participants, 38 completed both administrations of the GRASSP. Three participants were evaluated by different raters in trial 1 and trial 2 , and were therefore excluded from test-retest reliability analysis. Data on administration time were missing for three participants for trial one, and five participants for trial two. There were no differences in sample characteristics between trial one and trial two. 
Table 2 Mean, Standard Deviation and Range of GRASSP Values

\begin{tabular}{|c|c|c|c|c|c|c|c|c|c|c|c|c|c|c|c|}
\hline & \multicolumn{3}{|c|}{ Strength $(0-50)$} & \multicolumn{6}{|c|}{ Sensation (0-12) } & \multicolumn{6}{|c|}{ Prehension $(0-12)$} \\
\hline & \multirow[b]{2}{*}{ M } & \multirow[b]{2}{*}{ SD } & \multirow[b]{2}{*}{$\mathrm{R}$} & \multicolumn{3}{|c|}{ Dorsal } & \multicolumn{3}{|c|}{ Palmar } & \multicolumn{3}{|c|}{ Ability } & \multicolumn{3}{|c|}{ Performance } \\
\hline & & & & M & SD & $\mathrm{R}$ & M & SD & $\mathrm{R}$ & M & SD & $\mathrm{R}$ & M & SD & $\mathrm{R}$ \\
\hline $\mathrm{R}$ & 28.13 & 14.78 & $3-50$ & 8.70 & 4.62 & $0-12$ & 9.27 & 4.43 & $0-12$ & 6.17 & 4.66 & $0-12$ & 15.94 & 10.90 & $0-30$ \\
\hline $\mathrm{L}$ & 26.52 & 13.93 & $3-50$ & 8.78 & 4.50 & $0-12$ & 9.44 & 4.26 & $0-12$ & 5.79 & 4.47 & $0-12$ & 15.06 & 10.90 & $0-30$ \\
\hline
\end{tabular}

Mean (M), standard deviation (SD) and range (R) values for GRASSP subtests, trial one data $(n=47)$

$R$ right hand, $L$ left hand

Table 3 Mean, Standard Deviation, Range, and Median GRASSP Values for Motor Complete and Incomplete Injuries

\begin{tabular}{|c|c|c|c|c|c|c|c|c|c|c|c|c|c|c|c|c|c|c|c|c|c|c|}
\hline & & \multicolumn{7}{|c|}{ Strength $(0-50)$} & \multicolumn{14}{|c|}{ Prehension } \\
\hline & & \multirow[b]{2}{*}{ M } & \multirow[b]{2}{*}{ SD } & \multirow[b]{2}{*}{$\mathrm{R}$} & \multirow[b]{2}{*}{ MD } & \multirow[b]{2}{*}{ QR } & \multirow[b]{2}{*}{5 th } & \multirow[b]{2}{*}{95 th } & \multicolumn{7}{|c|}{ Ability (0-12) } & \multicolumn{7}{|c|}{ Performance $(0-30)$} \\
\hline & & & & & & & & & M & SD & $\mathrm{R}$ & MD & $\mathrm{QR}$ & 5 th & 95 th & M & SD & $\mathrm{R}$ & MD & QR & 5 th & 95th \\
\hline \multirow[t]{2}{*}{$\mathrm{C}$} & $\mathrm{R}$ & $20.83^{\mathrm{a}}$ & 13 & $3-50$ & 16.5 & 15 & 3 & 50 & $3^{\mathrm{a}}$ & 3.20 & $0-12$ & 3 & 5 & 0 & 12 & $11.22^{\mathrm{a}}$ & 8.78 & $0-30$ & 11.5 & 14 & 0 & 30 \\
\hline & $\mathrm{L}$ & 21.72 & 14.39 & $3-50$ & 16 & 24 & 3 & 50 & $3.44^{\mathrm{a}}$ & 3.57 & $0-12$ & 11.5 & 14 & 0 & 12 & 11.72 & 10.13 & $0-30$ & 14.5 & 18 & 0 & 30 \\
\hline \multirow[t]{2}{*}{ I } & $\mathrm{R}$ & $32.83^{\mathrm{a}}$ & 13.37 & $5-50$ & 33.5 & 25 & 5 & 50 & $8.67^{\mathrm{a}}$ & 3.73 & $0-12$ & 9 & 6 & 0 & 12 & $20.39^{\mathrm{a}}$ & 9.75 & $0-30$ & 25 & 17 & 0 & 30 \\
\hline & $\mathrm{L}$ & 28.17 & 14.08 & $4-50$ & 31 & 19 & 4 & 50 & $7.28^{\mathrm{a}}$ & 4.58 & $0-12$ & 8 & 10 & 0 & 12 & 17.44 & 11.05 & $0-30$ & 19 & 23 & 0 & 30 \\
\hline
\end{tabular}

Mean (M), standard deviation (SD), range (R), median (MD), Quartile Range (QR), and 5th and 95th percentile values for motor complete (C) ( $N$ $=18)$ and incomplete (I) $(n=18)$ injuries

$R$ right, $L$ left

${ }^{\text {a }}$ Statistically significant difference between motor complete and incomplete injuries

The mean time for GRASSP completion for trial one $(n$ $=44)$ and trial two $(n=33)$ was $38.41(R=14-75)$ and $33.85(R=15-55) \mathrm{min}$, respectively. Although there were no statistically significant differences in administration time across age groups, time requirements were longest for the youngest participants (mean time $=45 \mathrm{~min}$ ).

Mean, standard deviation, and range values for each of the GRASSP sub-tests for the entire sample $(N=47)$ are shown in Table 2 . There were statistically significant differences between motor complete and motor incomplete groups in right hand strength $(p=0.010)$, right $(p=0.043)$ and left $(p=0.033)$ hand sensation, right hand ability $(p=$ $<0.001)$ and performance $(p=0.01)$ prehension function, and left hand ability prehension function $(p=0.014)$ (Table 3, Figs. 1 and 2). There were no differences in GRASSP sub-scores among the age or NL groups.

As shown in Table 4, the GRASSP strength subtest score had moderate correlation with the SCIM-III total score $\left(r_{\mathrm{s}}\right.$ $=0.52)$ and the SCIM-III SC score $\left(r_{\mathrm{s}}=0.53\right)$, and strong correlation with the CUE-Q $\left(r_{\mathrm{s}}=0.82\right)$. Total GRASSP prehension performance was strongly correlated with SCIM-III total $\left(r_{\mathrm{s}}=0.68\right)$, SCIM SC $\left(r_{\mathrm{s}}=0.70\right)$ and CUE$\mathrm{Q}\left(r_{\mathrm{s}}=0.84\right)$. GRASSP sensation subtest scores showed weak correlations with all comparison measures. Right and left hand sensation and strength had moderate to strong

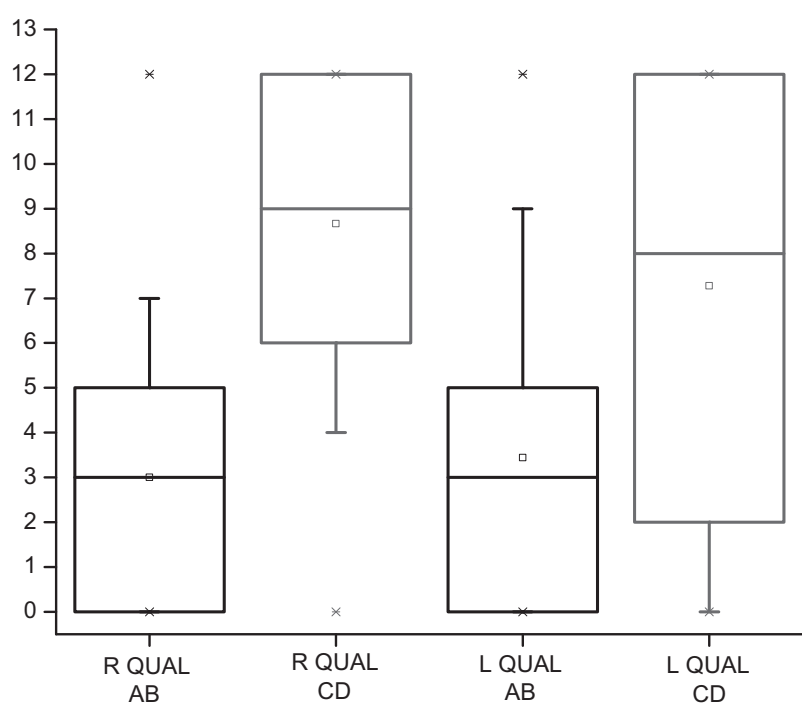

Fig. 1 Visual Representation of Distribution of Qualitative Prehension Ability Scores for Complete and Incomplete Injuries. Distribution of right $(\mathrm{R})$ and left $(\mathrm{L})$ hand qualitative prehension ability scores for complete $(\mathrm{AB})$ and incomplete injuries $(\mathrm{CD})$. The upper and lower margins of the box indicate the interquartile range, demarcating the 25 th and 75 th percentile. The center line is the medium (50th percentile), and the square within the box is the mean. The outer bars indicate range of scores at each end (5th and 95th percentile). The asterisks denote the lowest and highest score 


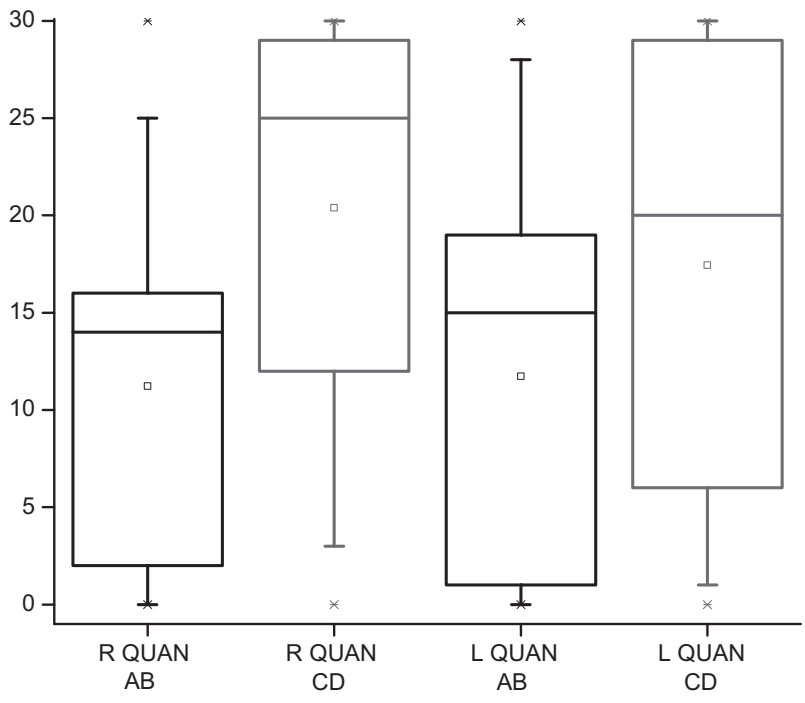

Fig. 2 Visual Representation of Distribution of Quantitative Prehension Ability Scores for Complete and Incomplete Injuries. Distribution of right $(\mathrm{R})$ and left $(\mathrm{L})$ hand quantitative prehension performance scores for complete $(\mathrm{AB})$ and incomplete $(\mathrm{CD})$ injuries. The upper and lower margins of the box indicate the interquartile range, demarcating the 25th and 75th percentile. The center line is the medium (50th percentile), and the square within the box is the mean. The outer bars indicate range of scores at each end (5th and 95th percentile). The asterisks denote the lowest and highest score

correlations with prehension performance (Table 5). Testretest reliability for each of the GRASSP subtests was strong for both the right and left hands. ICC values for all comparisons exceeded 0.950 . Similar findings of reliability were noted when examined for age, NL and AIS groups, with ICC ranging between 0.95 and 0.99 and lower confidence intervals ranging between 0.93 and 0.96 .

The GRASSP was administered to only a small number of participants within the youngest age group $(n=5,11 \%$ of total sample). There were three 3 year-olds, one 4 yearold and one 5 year-old. All 3 year-olds successfully completed each GRASSP subtest, two of whom completed both trials by the same administrator. The mean time for GRASSP administration to 3 year olds was $50(R=30-75)$ min. Due to the inability to understand and follow
Table 5 Strength of Association Between Prehension Performance and Hand Strength and Sensation

\begin{tabular}{|c|c|c|c|c|}
\hline \multirow[b]{2}{*}{ Right strength } & \multicolumn{2}{|c|}{$\begin{array}{l}\text { Right prehension } \\
\text { performance }\end{array}$} & \multicolumn{2}{|c|}{$\begin{array}{l}\text { Left prehension } \\
\text { performance }\end{array}$} \\
\hline & 0.88 & $p=0.01$ & & \\
\hline Right sensation & 0.56 & $p=0.001$ & & \\
\hline Left strength & & & 0.86 & $p=0.01$ \\
\hline Left sensation & & & 0.49 & $p=0.00$ \\
\hline
\end{tabular}

Pearson correlation $<0.60$ poor-to-fair; $0.60-0.89$ moderate and $=>0.90$ substantial

First Trial Data $(N=45)$

directions, the four year-old only completed the right and left prehension ability test, and four out of the six quantitative performance tasks with the left hand. The five yearold successfully completed all subtests on each side for both trials; administration time was $50 \mathrm{~min}$. The five children who failed the screening due to inability to follow directions due to age and development were 18 months and, 3, $4(n=$ $2)$, and 5 years of age. Each of the six year-olds $(n=3)$ successfully completed the GRASSP, with a high degree of reliability $(\mathrm{ICC}=0.99)$ and mean administration time of 38 $(R=26-60) \mathrm{min}$.

\section{Discussion}

The results of this study support the hypotheses that GRASSP scores have high test-retest reliability with repeated administration by trained raters, and, except for the sensory score, correlate well with SCIM-III and CUE-Q when administered to children. The finding of high reliability is consistent with other pediatric studies that report good reliability for manual muscle strength testing [24], sensory testing using the Semmes-Weinstein [25], and performance based measures [26, 27] in children.

The associations between GRASSP and SCIM-III total and SC scores, and the GRASSP and CUE-Q scores were similar to those reported in the adult literature [5], and

Table 4 Concurrent Validity of the GRASSP

\begin{tabular}{|c|c|c|c|c|c|c|}
\hline \multirow[b]{2}{*}{ GRASSP Sub Tests } & \multicolumn{3}{|c|}{ Pediatric sample } & \multicolumn{3}{|c|}{ Kalsi-Ryan et al. [5] } \\
\hline & $\begin{array}{l}\text { SCIM-III } \\
(N=26)\end{array}$ & $\begin{array}{l}\text { SCIM SC } \\
(N=26)\end{array}$ & $\begin{array}{l}\text { CUE-Q } \\
(N=36)\end{array}$ & $\begin{array}{l}\text { SCIM-III } \\
(N=72)\end{array}$ & $\begin{array}{l}\text { SCIM SC } \\
(N=72)\end{array}$ & $\begin{array}{l}\text { CUE-Q } \\
(N=72)\end{array}$ \\
\hline Sensation total $(\mathrm{R}+\mathrm{L})$ & 0.33 & 0.37 & $0.40^{\mathrm{a}}$ & 0.57 & 0.74 & 0.77 \\
\hline Strength total $(\mathrm{R}+\mathrm{L})$ & $0.52^{\mathrm{a}}$ & $0.53^{\mathrm{a}}$ & $0.82^{\mathrm{a}}$ & 0.59 & 0.74 & 0.76 \\
\hline $\begin{array}{l}\text { Prehension } \\
\text { performance total (R } \\
+\mathrm{L})\end{array}$ & $0.66^{\mathrm{a}}$ & $0.70^{\mathrm{a}}$ & $0.84^{\mathrm{a}}$ & 0.68 & 0.79 & 0.83 \\
\hline
\end{tabular}

Adult Sample is shown for comparison. Spearman correlation $<0.60$ poor-to-fair; $0.60-0.89$ moderate and $=>0.90$ substantial

${ }^{a}$ Except for sensation right and left, all values had significance level of $p<0.001$ 
provide evidence of concurrent validity of the GRASSP. Compared to adults [5], the pediatric GRASSP sensory score had lower correlation with SCIM-III total and SCIMIII SC scores, and the GRASSP strength score had lower correlation with SCIM-III SC. The smaller pediatric sample size may in part explain the weaker correlations. It is also plausible that weaker association between hand impairment (sensation and strength) and SCIM-III scores in children is a result of typical growth and development, where dependency upon parents (rather than hand function) for some activities of daily living (ADL) especially self-care is usual. Studies with larger sample sizes of young children with and without SCI are needed to examine the relationships among age, growth and development, ADL as measured by the SCIM-III and hand function as measured by the GRASSP. Nonetheless, the strong correlation between the GRASSP and CUE-Q corroborates the finding by Kalsi-Ryan [5] that the GRASSP is strongly associated with self-reported upper limb capabilities as measured by the CUE-Q.

The hypothesis that the GRASSP could distinguish between and across groups with known differences was not fully supported. While we anticipated that the GRASSP would discriminate across NL groups, we did not find differences in any GRASSP scores, likely due to small sample sizes. Small sample sizes prohibited stratification of each NL group by AIS. The GRASSP did however discriminate between motor complete and motor incomplete injuries in strength, sensation, and right hand prehension ability and performance, and left hand prehension ability. Given the strong correlation between strength and performance, the finding that both left hand strength and left hand performance did not discriminate between motor complete and incomplete injuries suggests that the two groups had similar left hand impairment level, and thus one would not expect the GRASSP to discriminate. Similarly, the small sample in this study prevented further stratification into NL groups within motor complete and incomplete categories. A larger sample size would enable more discrete groupings within motor complete and incomplete categories, and likely reduce the large variability seen in our results.

The finding that the GRASSP sensation and strength subtests did not discriminate across age groups was not unanticipated, and is consistent with published psychometric studies in pediatrics that show strength and sensation can be tested in young children, with a high level of reliability. As an example, Dua et al. [25] showed that children 6 years of age were able to participate in testing of two-point discrimination with a high degree of reliability that did not change with advancing age. Auld et al. [28]. recommended use of the Semmes-Weinstein monofilaments for pediatric practice and research based on work that showed strong test-retest reliability in young children with and without cerebral palsy. Likewise, in our own work on psychometric evaluation of the motor and sensory examinations of the ISNCSCI in children with SCI, we found a high degree of reliability on repeated administrations, and no differences in scores as a function of age [24].

Many of the more commonly used performance-based hand assessments evaluate hand dexterity and hand function by quantifying the number of objects manipulated over a certain period of time (e.g., speed, usually measured in seconds). Psychometric studies of timed-tests of hand function show that children's performance (speed) increases with advancing age, and does so linearly and incrementally with each year [29-31]. However, while the GRASSP requires manipulation of items within $75 \mathrm{~s}$, it is not a timed test, but rather is scored based on the degree to which mature pinch and grasp patterns are used to manipulate objects. The 75-s limit is set as the maximum time allocated for attempting to complete the task. While in-hand manipulation skills can continue to mature until 7 years of age [32], grasp and pinch patterns emerge between 12 months and 3 years of age, and are generally mature by six years of age [33]. Thus, differences across the three older age groups would not necessarily be expected.

While each of the GRASSP subtest was administered in this study to several children between three and five years of age, we do not recommend using them with children younger than six years old. We recommend the GRASSP sensory and strength subtests for children six years old and older, and the GRASSP prehension performance/ability subtest for children 8 years of age and older. The latter recommendation is based on normal development of intrinsic hand function and precise grasp and pinch which are not fully established until between 6 and 7 years of age [32-34], Thus, it would not be appropriate to administer the GRASSP - an instrument that evaluates mature grasp patterns - to children who may still be developing precision and in-hand manipulation skills. Importantly, despite our recommendation of 8 years old for the GRASSP prehension subtest(s), we acknowledge that there were only two 8 year olds and two 9 years olds who participated in this study. As such, practitioners and researchers who utilize the GRASSP prehension subtest with children should evaluate reliability in their own samples. Despite the challenges of measurement and the nuances of growth and development that must be considered when working with children, the GRASSP is a clinically relevant tool, despite the modest amount of time it requires to administer. Given the lack of robust hand measures for pediatric cSCI, information gained from administration of the GRASSP may be helpful in assessing individual patterns of recovery. A pediatric version of the GRASSP would be ideal, and include reduced administration time, child-friendly objects (not glass) and considerations of the developing hand while scoring. 
This study has limitations. Although geographically diverse, the sample was one of convenience and not representative of the pediatric SCI population. A larger sample with adequate distribution across ages, NL and AIS categories would be ideal and enhance the strength of these conclusions.

Despite strong evidence in support of the ISNCSCI in children six years of age and older [24], there was a relatively large number of participants without complete ISNCSCI examinations, and disallowed formal classification of NL and AIS. The majority of the ISNCSCI were missing in the youngest age group and is attributed to the inability to accurately perform the ISNCSCI in children younger than six years of age [24]. Also, if available we utilized ISNCSCI scores from the medical record if the examinations were performed within 12 months of study enrollment, but after neurological stability was documented. The rationale for the 12-month timeframe was based on usual standard of care that suggests conducting an annual neurological examination in children with SCI. Despite the chronicity of the study sample, it is possible that ISNCSCI scores obtained from the medical record were no longer accurate at the time of study enrollment. Moreover, we did not record the time between ISNCSCI examination and study enrollment, nor was obtaining the UE motor scores approved for this study. Future studies on the GRASSP would be strengthened by including these variables. The short time frame between the first and second administration could have potentially resulted in participants' recall or fatigue, both of which would influence the second administration, and the exact time between the two trials for each participant was not recorded. Most notably, the sample size was small and limited the sub-analyses that would help to establish psychometric properties of the GRASSP for various ages and for the four AIS categories. Finally, normative data for the prehension subtests are needed to aid interpretation when the GRASSP is used in children with cSCI. Responsiveness studies are needed prior to using the GRASSP in intervention studies with children. Data from this study could be used to determine sample size calculations for future work with children, which was not done for this study due to the void in pilot data or pediatric experience with the GRASSP.

\section{Conclusion}

This study has shown utility and provided initial psychometric evidence in support of the GRASSP with youth with cSCI. We recommend using the GRASSP strength and sensory subtests with children who are six years of age and older, and the GRASSP prehension performance/ability subtests with children who are 8 years of age and older. The study findings demonstrate good reliability of the GRASSP upon repeated measures by the same trained administrator. The findings also demonstrated concurrent and knowngroup validity of the GRASSP.

Acknowledgements This study was funded by the Craig $\mathrm{H}$ Neilsen Foundation, Spinal Cord Injury Research on the Translation Spectrum, Senior Research Award \#282592 (Mulcahey, PI). Becky Sinko OTD, OTR/L, Jennifer L Silvestri, MS, OTR/L, Lauren White, PT, DPT, PCS, Julie A Cagney PT, DPT, PCS, Ingrid Parry, PT, Jennifer Schottler MSPT, Kristine Higgins, PT, MS, NCS, Michelle L. Feltz, BA, and Jenny Mazurkiewicz MS, OTR/L are acknowledged for their role in data collection. Sue Duff is acknowledge for review of the manuscript.

Funding The study was funded by the Craig H. Neilsen Foundation, Spinal Cord Injury Research on the Translation Spectrum, Senior Research Award Grant \#282592 (Mulcahey, PI).

\section{Compliance with ethical standards}

Conflict of interest Dr. Mulcahey receives research support from the Shriners Hospitals for Children, National Institute Neurological Disease and Stroke, The Craig H. Neilsen Foundation and The Rick Hansen Institute. She receives compensation for her role as Associate Editor for Topics in Spinal Cord Rehabilitation, royalties for the textbook "The Child and Young Adult with Spinal Cord Injury" and has received compensation as review panel chair/member for the Dept. of Defense and the Craig H. Neilsen Foundation. Dr. Vogel receives compensation for his role as Editor for Topics in Spinal Cord Injury Rehabilitation, royalties for the textbook "The Child and Young Adult with Spinal Cord Injury" and has received compensation as review panel chair/member for the Dept. of Defense. The remaining authors declare no conflict of interest.

\section{References}

1. Kalsi-Ryan S, Curt A, Fehlings MG, Verrier MC. Assessment of the hand in tetraplegia using the graded redefined assessment of strength, sensibility and prehension (GRASSP): impairment versus function. Top Spinal Cord Inj Rehabil. 2009;14:34-46.

2. Weinstein S. Fifty years of somatosensory research: from the Semmes-Weinstein monofilaments to the Weinstein Enhanced Sensory Test. J Hand Ther. 1993;6:11-22.

3. Kirshblum S, Waring W. Updates for the International Standards for Neurological Classification of Spinal Cord Injury. Phys Med Rehabil Clin N Am. 2014;25:505-17.

4. Sollerman C, Ejeskär A. Sollerman hand function test. A standardised method and its use in tetraplegic patients. Scand J Plast Reconstr Surg Hand Surg. 1995;29:167-76.

5. Kalsi-Ryan S, Beaton D, Curt A, Duff S, Popovic MR, Rudhe C, et al. The graded redefined assessment of strength, sensibility and prehension: reliability and validity. J Neurotrauma. 2012;29:905-14. 1089/neu.2010.1504

6. Kalsi-Ryan S, Curt A, Verrier MC, Fehlings MG. Development of the graded redefined assessment of strength, sensibility and prehension (GRASSP): reviewing measurement specific to the upper limb in tetraplegia. J Neurosurg: Spine. 2012;12(Suppl):65-76.

7. Velstra IM, Bolliger M, Krebs J, Rietman JS, Curt A. Predictive value of upper limb muscles and grasp patterns on functional outcome in Cervical Spinal Cord Injury. Neurorehabil Neural Repair. 2016;30:295-306. 10.1177/1545968315593806. 
8. Zariffa J, Curt A, Verrier MC, Fehlings MG, Kalsi-Ryan S. GRASSP Cross-Sectional Study Team and Ontario GRASSP Longitudinal Study Team. Predicting task performance from upper extremity impairment measures after cervical spinal cord injury. Spinal Cord. 2016;54:1145-51. 10.1038/sc.2016.77.

9. Kalsi-Ryan S, Beaton D, Ahn H, Askes H, Drew B, Curt A, et al. Responsiveness, sensitivity, and minimally detectable difference of the graded and redefined assessment of strength, sensibility, and prehension, Version 1.0. J Neurotrauma. 2016;33:307-14. https:// doi.org/10.1089/neu.2015.4217

10. Velstra IM, Curt A, Frotzler A, Abel R, Kalsi-Ryan S, Rietman JS, et al. Changes in strength, sensation, and prehension in acute cervical spinal cord injury: European Multicenter Responsiveness Study of the GRASSP. Neurorehabil Neural Repair. 2015;29:755-66. https://doi.org/10.1177/1545968314565466

11. Zariffa J, Kapadia N, Kramer JLK, Taylor P, Alizadeh-Meghrazi M, Zivanovic V, et al. Relationship between clinical assessments of function and measurements from an upper limb robotic rehabilitation device in cervical spinal cord injury. IEEE Trans Neural Syst Rehabil Eng. 2012;20:341-50.

12. Zariffa J, Kapadia N, Kramer JLK, Taylor P, Alizadeh-Meghrazi M, Zivanovic V, et al. Feasibility and efficacy of upper limb robotic rehabilitation in subacute cervical spinal cord injury population. Spinal Cord. 2012;50:220-6.

13. Zbogar D, Eng JJ, Miller WC, Krassioukov A, Verrier MC. Movement repetitions in physical and occupational therapy during spinal cord injury rehabilitation. Spinal Cord. 2017;55:172-9.

14. Sinnott KA, Dunn JA, Wangdell J, Johanson ME, Hall AS, Post MW. Measurement of outcomes of upper limb reconstructive surgery for tetraplegia. Arch Phys Med Rehab. 2016;96(suppl 2): S169-82.

15. Mulcahey MJ, Weiss A. Contemporary issues related to management of the upper limb in tetraplegia. Hand Clin. 2008;24:xi.

16. Kozin S. Tetraplegia. J Hand Surg. 2002;2:141-52.

17. Betz RR, Mulcahey MJ, Smith BT. Unique aspects of the pediatric patient with spinal cord injury and the effects on upperlimb management. J Rehabil Res Dev. 2004;41:15.

18. Mulcahey MJ, Betz RR, Smith BT, Weiss AA. A prospective study of the outcomes of tendon transfers with children with tetraplegia. J Pediatr Orthop. 1999;19:319-28.

19. Mulcahey MJ, Zlotlow D, Kozin S. Upper extremity management. In: Vogel, Zebracki, Betz RR and Mulcahey MJ eds. The Child With SCI, 2nd edition. McKeith Press London; 2014. ISBN:9781-909962-34-7.

20. Biering-Sorensen F, Alai S, Anderson K, Charlifue S, Chen Y, DeVivo M, et al. Common data elements for spinal cord injury clinical research: a National Institute for Neurological Disorders and Stroke project. Spinal Cord. 2015;53:265-77.
21. Catz A, Itzkovich M, Agranov E, Ring H, Tamir A. SCIM-spinal cord independence measure: a new disability scale for patients with spinal cord lesions. Spinal Cord. 1997;35:850-6.

22. Mulcahey MJ, Thielen CC, Sadowsky C, Silvestri JL, Martin R, White L, et al. Despite limitations, the SCIM-III is reproducible and a valid indicator of physical function in youth with SCI. Spinal Cord. 2017. https://doi.org/10.1038/s41393-017-0036-0

23. Oleson CV, Marino RJ. Responsiveness and concurrent validity of the revised capabilities of the upper extremity questionnaire (CUEQ) in patients with acute tetraplegia. Spinal Cord. 2014;52:625-8.

24. Mulcahey MJ, Vogel L, Betz R, Samdani A, Chafetz R, Gaughan $\mathrm{J}$. The international standards for neurological classification of spinal cord injury: psychometric evaluation and guidelines for use with children and youth. Phys Med Rehabil. 2011;92:1264-9. https://doi.org/10.1016/j.apmr.2011.03.003

25. Dua K, Lancaster TP, Abzug JM. Age-dependent reliability of Semmes-Weinstein and 2-point discrimination tests in children. J Pediatr Orthop. 2016. https://doi.org/10.1097/BPO. 0000000000000892

26. Reedman SE, Beagley S, Sakzewski L, Boyd RN. The Jebsen Taylor Test of Hand Function: a pilot test retest reliability study in typically developing children. Phys Occup Ther Pediatr. 2016;36:292-304.

27. Mulcahey MJ, Smith BT, Betz RR. Psychometric rigor of the grasp and release test for measuring functional limitation of persons with tetraplegia: a preliminary analysis. Spinal Cord. 2004;27:41-46.

28. Auld ML, Ware RS, Boyd RN, Moseley GL., Johnston LM. Reproducibility of tactile assessment for children with unilateral cerebral palsy. Phys Occup Ther Pediatr. 2012 32:151-66.

29. Gogola G, Velleman PF. Hand dexterity in children: administration and normative values of the functional dexterity test. J Hand Ther. 2013;38:2426-31.

30. Smith Y, Hong E, Presson C. Normative validation studies of the nine hole peg test with children. Percept Mot Skills. 2000;90:823-4.

31. Beagley SB, Reedman SE, Sakzewski L. Establishing Australia norms for the Jebsen Taylor test of hand function in typically developing children aged 5-10 years: a pilot study. Phys Occup Ther Pediatr. 2016;36:88-109.

32. Humphrey R, Jeweel K, Rosenberger RC. Development of inhand manipulation and relationship with activities. Am J Occup Ther. 1995;49:763-71.

33. Exner C. Development of hand skills. In: Case-Smith J, editor. Occupational therapy for children. 5 th ed. St Louis: Elsevier, Inc; 2005.

34. Mathiowetz V, Wiemer DM, Federman SM. Grip and pinch strength: norms for 6-to 19-year olds. Am J Occuptional Ther. 1986;140:705-11. 